\title{
CONCEPCIONES EN TORNO A LA DEMOCRACIA Y LA CIUDADANÍA DE LOS ESTUDIANTES DE LA UNIVERSIDAD SANTO TOMÁS - SECCIONAL BUGARAMANGA'
}

(Recibido: Junio 12 de 2012 Aprobado: Julio 10 de 2012)

\author{
Roberto Alonso Cardona Ospina ${ }^{2}$ \\ Gilberto Bonilla Sánchez ${ }^{3}$ \\ Dénix Alberto Rodríguez Torres ${ }^{4}$
}

\section{Resumen}

El artículo contiene el análisis de los datos recolectados a través de la aplicación de instrumentos propios de los enfoques cuantitativo y cualitativo, que permitieron determinar las concepciones sobre la ciudadanía de los estudiantes de la Universidad Santo Tomás, Bucaramanga; estudio realizado en el marco del grupo de Investigación en Desarrollo Humano, en la línea de investigación de Estudios Políticos, que lidera el Departamento de Humanidades . Además, ofrece los datos estadísticos y descriptivos que se analizaron a la luz del marco teórico de referencia, la metodología y los hallazgos obtenidos. Los resultados derivados del análisis hecho a los datos recolectados, son presentados como aportes reflexivos y como primeras conclusiones a la investigación desarrollada sobre: la política, la democracia y la ciudadanía, macrocategorías de referencia del proyecto.

\section{CONGEPTIONS ON DEMOGRACY AND GITIZENSHIP OF STUDENTS AT THE UNIVERSITY SANTO TOMÁS - BUGARAMANGA}

\begin{abstract}
Alostract
This article contains the analysis of the data collected through the application of instruments of quantitative and qualitative approach that allowed determining the conceptions about the citizenship of the students at the University Santo Tomás, Bucaramanga; the study was carried out within the framework of the Group of Research in Human Development, in the research line of Political Studies, lead by the Department of Humanities. It also provides the statistical and descriptive data that were analyzed in the light of the theoretical framework of reference, the methodology and the findings obtained. The results of the analysis of the collected data are presented as thoughtful contributions and initial findings to the research carried out on: politics, democracy and citizenship, the project reference macro-categories.
\end{abstract}

\section{Kev words}

Democracy, Citizenship, Policy.

1 Artículo producto de la Investigación Institucional realizada por el grupo de investigación En Desarrollo Humano, perteneciente al Departamento de Humanidades de la Universidad Santo Tomás de Bucaramanga, en la línea de Estudios Políticos.

2 Licenciado en Filosofía de la Universidad Minuto de Dios, Bogotá, (1996). Especialista en Docencia Universitaria de la Universidad Santo Tomás, Bogotá (2000). Diplomado en Manejo de Ambientes Virtuales para el Aprendizaje de la Universidad Santo Tomás, Bucaramanga (2008). Magíster en Pedagogía de la Universidad Industrial de Santander, (2009). Diplomado en Ética de la Investigación, Universidad Santo Tomás, Bucaramanga, (2010) Docente Investigador en la Universidad Santo Tomás, Bucaramanga. www.roalcaos.blogspot.com

3 Magíster en Pedagogía de la Universidad Industrial de Santander, (2010), Filósofo de la Universidad Industrial de Santander, (2005), Diplomado en Manejo de Ambientes Virtuales para el Aprendizaje de la Universidad Santo Tomás, Bucaramanga (2010) Docente Investigador Universidad Santo Tomás, Bucaramanga. gbonillasan@hotmail.com

4 Licenciado en Filosofía, Universidad Minuto de Dios, Bogotá (1996); Estudios de Teología en la Universidad Javeriana, Bogotá (2006); Diplomado en Ambientes Virtuales, Universidad Santo Tomás, Bucaramanga. (2008) Magíster En Educación (VUAD), 2010. Doctorando en Educación (2012) Diplomado en ética de la Investigación. Universidad Santo Tomás, Bucaramanga (2010). Docente Investigador Universidad Santo Tomás. Tutor Universidad Abierta y a Distancia (VUAD). denixvirtual@gmail.com 


\section{Introducción}

Reflexionar sobre la ciudadanía se ha convertido en una necesidad que las sociedades contemporáneas han tratado de suplir de diversas maneras. Una de ellas ha sido a través de la investigación de las prácticas, concepciones y percepciones que tienen los individuos sobre dicho tema. En el marco del proyecto de investigación institucional denominado: “Concepciones en torno a la democracia y la ciudadanía de los estudiantes de la Universidad Santo Tomás -Bucaramanga", dentro de la línea de investigación sobre Estudios Políticos, hemos tratado de contribuir y participar de la reflexión y discusión y aportar desde la academia elementos que han resultado de las investigaciones realizadas sobre el tema. En una ocasión anterior se compartió una aproximación teórica sobre el concepto de ciudadanía ${ }^{5}$ que condesaba las reflexiones ofrecidas por reconocidos intelectuales que, desde la economía, la filosofía y la pedagogía amplían la discusión hacia la ciudadanía planetaria. En esta ocasión, compartimos elementos fundamentales del proyecto como: los objetivos, la metodología utilizada para desarrollar y alcanzar los objetivos del proyecto y la sistematización de los datos recolectados a través de los instrumentos utilizados en el estudio, la descripción de los resultados estadísticos de las doce categorías conceptuales abordadas, el análisis de las tendencias y frecuencias que se presentaron en cada uno de los ítems que conforman la encuesta y algunas conclusiones y recomendaciones que surgen como resultado de este tipo de indagación.

\section{Contextualización}

El proyecto "Concepciones en torno a la democracia y la ciudadanía de los es-

5 "Del concepto de Ciudadanía: una aproximación teórica". Revista Temas, Vol. III, Número 5, Octubre de 2011. Departamento de Humanidades, Universidad Santo Tomás, Bucaramanga. Pp. 93-110 tudiantes de la Universidad Santo Tomás - Bucaramanga" en su primera fase está orientado al diagnóstico de las concepciones en torno a la democracia y la ciudadanía en los estudiantes de la Universidad. Se propuso la exploración y comprensión de los imaginarios de los estudiantes de la Universidad frente a estos temas para, en una fase posterior de la investigación, crear espacios de formación donde los estudiantes mejoren sus concepciones y prácticas en torno a la ciudadanía, a fin de enriquecer la formación humanística que brinda la Universidad y contribuir a la construcción de un modelo de formación ciudadana para la USTA.

El problema abordado, se condensa en la pregunta:¿Cuáles son las percepciones, actitudes y concepciones en torno a la democracia y la ciudadanía en los estudiantes de la Universidad Santo Tomás - Bucaramanga? que en todo momento sirvió de brújula para las diferentes exploraciones que se realizaron, con el fin de alcanzar los objetivos propuestos para la primera fase del estudio.

\section{Metodología}

El enfoque de investigación que se utilizó fue predominantemente mixto, con la utilización de una técnica cuantitativa (La encuesta) y una técnica cualitativa (los grupos de discusión o grupos focales). En consonancia con lo expresado por algunas teorías contemporáneos sobre metodología de investigación social y la tendencia a combinar los métodos y las técnicas cuantitativas y cualitativas.

Como instrumento de recolección de información se utilizó el "Cuestionario sobre Democracia, Cultura Política y Ciudadanía (conceptos y actitudes)"elaborado por el Dr. Ángel Villarini Jusino. Este cuestionario tiene como propósito recopilar información que permita conocer las percepciones, ideas o conceptos y actitudes que tienen los estudiantes a los 
cuales se les administra, en relación con la política, la democracia y la ciudadanía.

El instrumento contiene 60 ítems de selección múltiple, 6 sobre datos generales de los estudiantes y 54 ítems organizados en torno a 12 categorías fundamentales relacionadas con la democracia, la política y la ciudadanía. La Encuesta fue digitalizada e incluida en las aulas virtuales de la plataforma Moodle, de los programas de Humanidades que cursan los estudiantes de las diferentes facultades.

Los doce conceptos, o categorías en mención, son: política, poder político, Estado, gobierno, democracia, ciudadanía democrática, sistema de derecho, sistema representativo electoral, partido político, ideología, sociedad civil y clase social, hacen parte de tres macro-categorías (Política, Democracia y Ciudadanía) sobre las que giró el análisis que se presenta a continuación.

\section{Resultados de la investigación}

El siguiente análisis, como se mencionó anteriormente, se hace a partir de la tabulación del total de respuestas ofrecidas por los estudiantes con respecto a la cultura política, la democracia y la ciudadanía enmarcados en tres macro-categorías propias de la temática; sólo se mencionan las opciones que obtuvieron mayor porcentaje de selección y las menos preferidas por los encuestados ${ }^{6}$, de esta forma, a través de la interpretación del lenguaje estadístico por medio del teórico se obtiene la siguiente información:

\section{Caracterización de la población}

La población que participó del estudio a través del instrumento aplicado suministró los siguientes datos con respecto a sus características socio-demográficas:

6 Una información ampliada y detallada de la presente investigación, se puede consultar en el informe final que condensa todos los resultados del estudio y se encuentra en proceso de publicación por parte de la Universidad Santo Tomás, Bucaramanga, con proyección a Diciembre de 2012.
- Los estudiantes se clasificaron según el género, el estrato socioeconómico y la edad de la siguiente forma:

Tabla 1. Porcentajes de Edad

\begin{tabular}{cc}
\hline RANGO DE EDAD & PORCENTAJE \\
\hline Entre 18 y 22 años & $93 \%$ \\
Entre 23 y 27 años & $5 \%$ \\
Entre 28 y 32 años & $1 \%$ \\
Entre 33 y 40 años & $1 \%$ \\
40 o más años & $0 \%$ \\
\hline
\end{tabular}

Tabla 2. Porcentaje de Género

\begin{tabular}{cc}
\hline GÉNERO & PORCENTAJE \\
\hline Masculino & $32 \%$ \\
Femenino & $68 \%$ \\
\hline
\end{tabular}

Tabla 3. Porcentajes por grupo Social-Estrato Socio-Económico

\begin{tabular}{cc}
\hline GRUPO SOCIAL & PORCENTAJE \\
\hline Alto & $2 \%$ \\
Medio alto & $22 \%$ \\
Medio & $63 \%$ \\
Medio bajo & $12 \%$ \\
Bajo & $1 \%$ \\
\hline
\end{tabular}

Se puede afirmar, entonces, que la muestra del estudio se caracteriza por ser en su mayoría mujeres entre los 18 y 22 años de edad pertenecientes al grupo social o estrato socio-económico medio de la población. 


\section{Análisis descriptivo de las Macro-categorías conceptuales}

Gráfica 1. Política

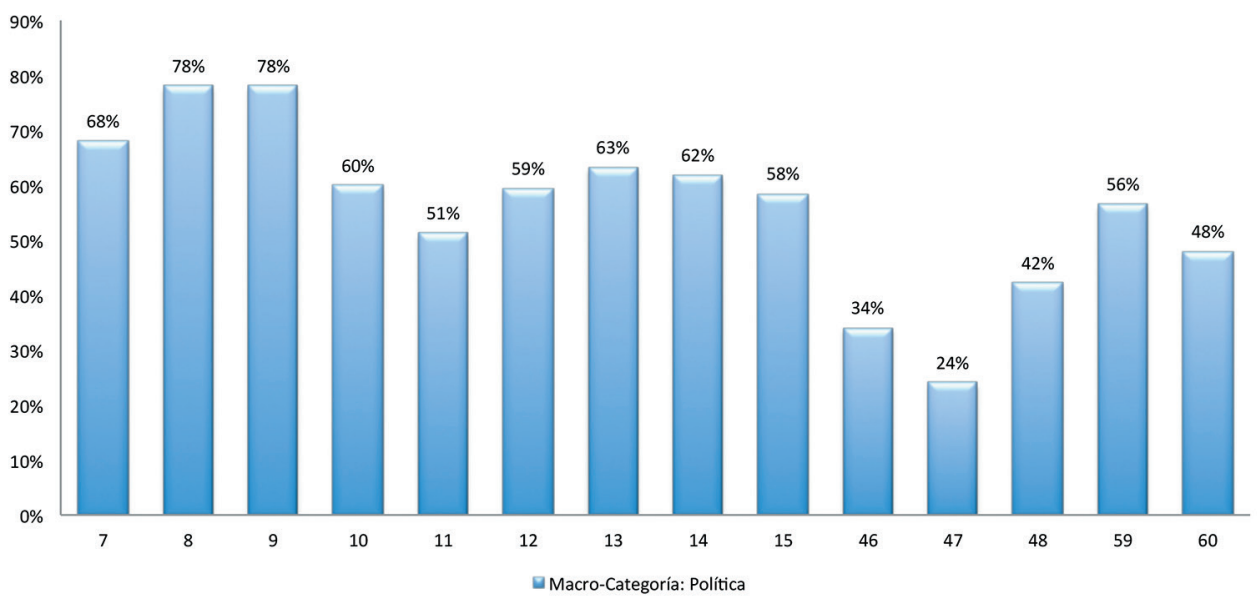

Al preguntar a los estudiantes sobre la idea que mejor resume lo que es la política, las tendencias de selección se inclinaron en un $68 \%$, que consideran que la política es el arte de gobernar para el bien de una colectividad y para el $23 \%$ la política es, las actividades y la competencia entre los partidos políticos.

Sobre la idea que mejor resume lo que en la práctica es la política en el país, el $78 \%$ opina que es una actividad para el beneficio de unos pocos y sólo el $13 \%$ opina que es un espectáculo o deporte nacional.

Cuando se exponen diferentes afirmaciones sobre la política a los estudiantes, y se indaga con cuál de ellas están de acuerdo, el 78\%manifiesta estar de acuerdo con la idea que expresa que se debe luchar para cambiar la forma en que se practica la política en nuestra sociedad; el $16 \%$ de los estudiantes en cambio, considera que lo mejor que los individuos pueden hacer, es no meterse en asuntos de política y que cada cual se ocupe de lo suyo.

Aunque la política no debería ser entendida o calificada desde concepciones morales, al relacionar la política con la moral el $60 \%$ cree que ésta es moral e inmoral según los fines que persiguen los que la llevan a cabo, mientras que el $21 \%$ dice que la política en ocasiones es moral y en otras no lo es, pues el fin justifica los medios.

El $51 \%$ considera que si existiera un movimiento, grupo o partido político de personas honestas y que defendiera causas justas, estaría dispuesto a participar de sus actividades; sólo el $3 \%$ dice que la política les interesa lo mínimo, como ir a votar cada cuatro años.

Respecto al rol de la Universidad frente a la situación política actual del país, el $59 \%$ cree que la Universidad debe llevar a cabo estudios que ayuden a orientar a la ciudadanía para la toma de decisiones políticas. Por el contrario, sólo el $13 \%$ considera que esta institución no debe intervenir o participar en ningún asunto político.

El $63 \%$ opina que el poder político es la fuerza o facultad que una comunidad o pueblo le otorga a una persona o grupo de personas para gobernarlos; el 6\% de los estudiantes dice que es la capacidad de usar la violencia para controlar a otros.

Lo que le concede poder político a una persona o grupo para dirigir el país es para el $62 \%$ el respaldo que le otorgue un partido político, y solo el $15 \%$ consi- 
dera que el poder político se obtiene con carisma o personalidad seductora.

A la pregunta qué grupo o institución tiene mayor poder político en el país, el $58 \%$ respondió que son los empresarios; el $28 \%$ cree que son los medios de comunicación; el $3 \%$ opina que es la Iglesia; el $3 \%$ considera que los pobres y el $7 \%$ los trabajadores.

Para la mayoría de los estudiantes la idea que mejor expresa lo que en la práctica es un partido político, según el $34 \%$, una agrupación de ciudadanos que se organiza en torno a un ideal para construir una sociedad mejor, y el $4 \%$ piensa que es un grupo de ciudadanos que busca defender sus intereses personales.

Para el $24 \%$ los partidos políticos fortalecen la democracia porque representan diferentes puntos de vista sobre lo que le conviene al pueblo. El 14\% piensa que debilitan la democracia porque limitan el número de puntos de vista y opciones sobre lo que le conviene al pueblo.

El $42 \%$ no simpatiza con partido alguno; y solo el $3 \%$ de los estudiantes manifiesta ser miembro de uno de los partidos de nuestra sociedad.

Gráfica 2. Democracia

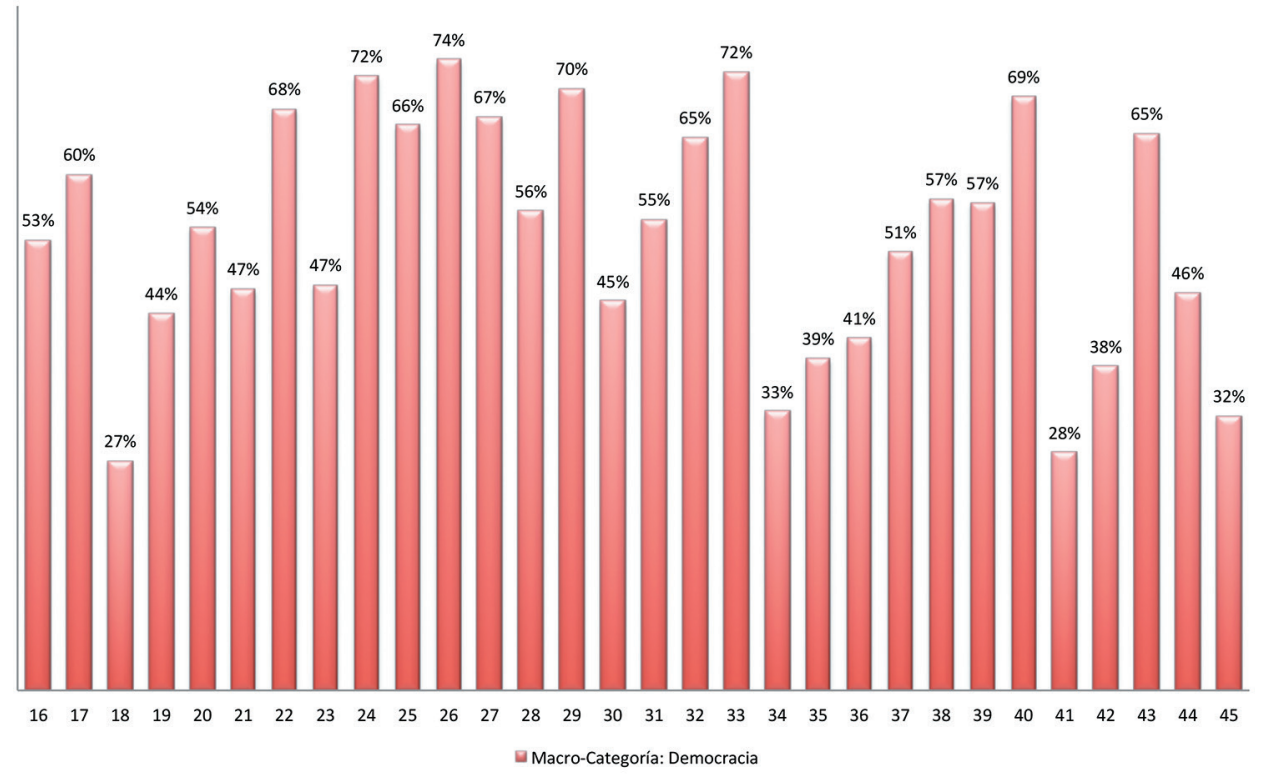

El $53 \%$ de los estudiantes opina que la más importante característica de la democracia como sistema político es que el pueblo o los ciudadanos seleccionen libremente a los que los representan en el gobierno. El $10 \%$ piensa que otra característica es que el pueblo o los ciudadanos tienen libertad de expresión y asociación.

El $60 \%$ cree que para que pueda haber efectivamente democracia en un país, es necesario que haya un sistema de derecho (Leyes) que garantice que el poder esté en manos del pueblo. El 59\% cree que el pueblo debe educarse para que pueda ejercer el poder y el $17 \%$ opina que debe existir un sistema de múltiples partidos.

El $27 \%$ cree que la finalidad más importante en un sistema político democrático es el respeto a los derechos civiles de todos los ciudadanos, mientras que solo el $8 \%$ de los estudiantes opina que la finalidad es el respeto a la propiedad privada de cada ciudadano. 
El $44 \%$ cree que el sistema político que impera en el país es democrático, pero tiene mucho que mejorar; solo el $6 \%$ considera que es perfectamente democrático.

El $54 \%$ considera al Estado como la principal institución de un sistema político, porque tiene los mecanismos de vigilancia, control y represión para velar por mantener el orden y garantizar la seguridad interna y externa del país. Solo el $12 \%$ considera es el responsable de la defensa nacional.

El $47 \%$ cree que el Estado es la estructura de poder dentro de un territorio, mientras que el gobierno es solo una parte del Estado; el $11 \%$ cree que el Estado y el Gobierno son el mismo.

El $68 \%$ piensa que una característica del Estado en un Sistema Político Democrático es el respeto a los derechos establecidos en una Constitución. Para el $16 \%$ el Estado está integrado por partidos políticos.

Históricamente el Estado democrático ha incurrido en algunas degeneraciones para el $47 \%$ el Estado se ha convertido en un aparato de represión al servicio de los intereses de unos grupos sociales o partidos políticos; un $32 \%$ opina que el Estado puede llegar a traspasar sus límites e intervenir en aspectos de la vida privada de los ciudadanos.

El $72 \%$ cree que para evitar que el Estado Democrático degenere, lo más conveniente sería educar al ciudadano para proteger su poder y sus derechos frente al Estado, y solo el $10 \%$ cree que se debería eliminar al Estado sustituyéndolo por un Gobierno sin Estado.

El $66 \%$ cree que la función de la Constitución en un sistema democrático es establecer y proteger los derechos de los ciudadanos y garantizar el debido proceso de Ley. El 16\% cree que es dar- le legitimidad a la distribución del poder que ocurre al crearse el Sistema Político.

El $74 \%$ respondió que el principio de igualdad supone que todo el mundo tiene los mismos Derechos Humanos y civiles, y el $15 \%$ supone que todos somos iguales porque somos hijos de Dios.

En una democracia el sistema de derechos es justo según el $67 \%$ si todos los ciudadanos son tratados como iguales ante la ley; solo el $14 \%$ entiende la justicia del sistema como el derecho que tiene toda persona de hacer lo que quiera.

El $56 \%$ supone que la libertad consiste en que cada ciudadano tiene derechos civiles fundamentales, como los de expresión, creencia y asociación. Solo el $16 \%$ cree que cada ciudadano puede hacer lo que quiera sin que nadie interfiera con él o ella.

El $70 \%$ cree que en un sistema democrático las leyes deben ser hechas en consulta con el pueblo. Solo el $11 \%$ cree que las leyes deben ser obedecidas no importa lo que digan o cómo fueron aprobadas.

Para el $45 \%$ un sistema democrático de Derecho debe incluir el respeto y la protección de los derechos civiles, humanos y ambientales o ecológicos y culturales; y el $6 \%$ cree que debe incluir el respeto a los derechos ambientales o ecológicos.

Según el $55 \%$ en un sistema de Derecho democrático la voluntad de la mayoría expresada legalmente, es la que manda, pero dentro de los límites que establece la constitución del país, y el $4 \%$ manifiesta que no cuenta si esa mayoría no está debidamente educada.

Con respecto al sistema de Derecho del país, el $65 \%$ piensa que a algunas personas o grupos se le respetan más 
sus derechos que a otros, y solo el $8 \%$ cree que hay demasiados derechos.

El $72 \%$ cree que un buen gobierno es aquel que hace respetar y respeta los derechos humanos, civiles y sociales de los ciudadanos, y solo el $19 \%$ lo identifica con el gobierno eficiente y efectivo en el control de los gastos.

Del gobierno democrático, el 33\% de los encuestados piensa que es preferible un gobierno autoritario, con gente que sepa gobernar, que pueda resolver de forma eficiente y efectiva los problemas del país, y el $6 \%$ opina que le es indiferente una u otra forma de gobierno.

Con respecto a la presente estructura de gobierno en el país, el 39\% opina que las tres ramas del poder público deben tener igual poder. Solo el $11 \%$ cree que el presidente o gobernador debe tener más poder del que tiene actualmente.

El $41 \%$ cree que el partido y el gobierno son distintos, pues el partido representa el modo de ver y los intereses de un sector, pero el gobierno es de todo el pueblo y solo el $4 \%$ cree que los partidos y el gobierno son lo mismo, pues son las mismas personas.

Frente a la relación entre gobierno democrático y medios de comunicación, el $51 \%$ cree que el gobierno no debe tener control alguno sobre los medios, salvo en aquellas situaciones definidas por la ley en que esté en peligro la seguridad del País o de los ciudadanos; solo el $13 \%$ cree que el gobierno se debe limitar a exhortar a que sean los propios medios los que se censuren cuando sea necesario.

De la relación entre gobierno democrático y las personas o familias, el $57 \%$ considera que un gobierno tiene derecho a prohibir el fumar en lugares públicos. Solo el $25 \%$ opina que el gobierno tiene derecho a decretar el servicio militar obligatorio.
Entre los principales problemas de gobernabilidad que se presentan en los sistemas políticos democráticos, según el $57 \%$, se encuentra, la pérdida de confianza en los "políticos" (líderes, partidos, burócratas) por parte del pueblo; el $26 \%$ piensa que otro problema es el clientelismo y la dependencia del Estado y, la misma cantidad piensa que la exclusión y marginalización de minorías, ilegales e indocumentados, entre otros es el principal problema de gobernabilidad.

Para el $69 \%$ la forma de atender los problemas de gobernabilidad que presentan los sistemas políticos democráticos es necesario implantar por medio de leyes e instituciones la educación, formación, capacitación ciudadana y conciencia, y solo el $15 \%$ cree que se debe implantar la descentralización y la redistribución del poder.

Para el $28 \%$ la idea que, en teoría, mejor expresa lo que es el sistema representativo electoral es la que permite que el pueblo elija unos representantes que son sus servidores y cuyos poderes están establecidos por ley. Solo el 7\% considera que el pueblo delega su poder en sus representantes, que gobiernan en su nombre.

Se argumenta que aunque la democracia directa sería la más perfecta porque el pueblo ejercería el poder directamente, sin intermediarios, eso no es posible por el tamaño y complejidad de nuestra sociedad. Esta afirmación es cierta para un $38 \%$ porque si cuesta trabajo que la gente vaya a votar, sería imposible que esté dispuesta a reunirse constantemente a deliberar. Sólo el 7\% considera que es cierto porque la mayoría de personas no tienen interés en la participación política.

En teoría el sistema representativo electoral es apropiado para la democracia siempre y cuando las personas 
electas, según el $65 \%$ estén capacitadas moral e intelectualmente para el arte de gobernar. El $22 \%$ cree que las personas electas pueden ser destituidas sino representan adecuadamente la voluntad de sus electores.

El $46 \%$ de los participantes, piensa que en Colombia la mayoría de las personas que elegimos como nuestros representantes, son electas porque saben cómo venderse a través de campañas de publicidad, y el $30 \%$ piensa que los representantes son elegidos porque han sido seleccionados por un partido, aunque no sean las más capacitadas.

Según la experiencia histórica y la realidad presente en mi país, el Sistema Representativo Electoral según el 32\% tiene que mejorarse mediante un procedimiento, que no sea el de los partidos políticos, para seleccionar a las personas moral e intelectualmente más capacitadas para gobernarnos; y el $11 \%$ cree que el Sistema Representativo Electoral debe permanecer tal cual es.

Gráfica 3. Ciudadanía

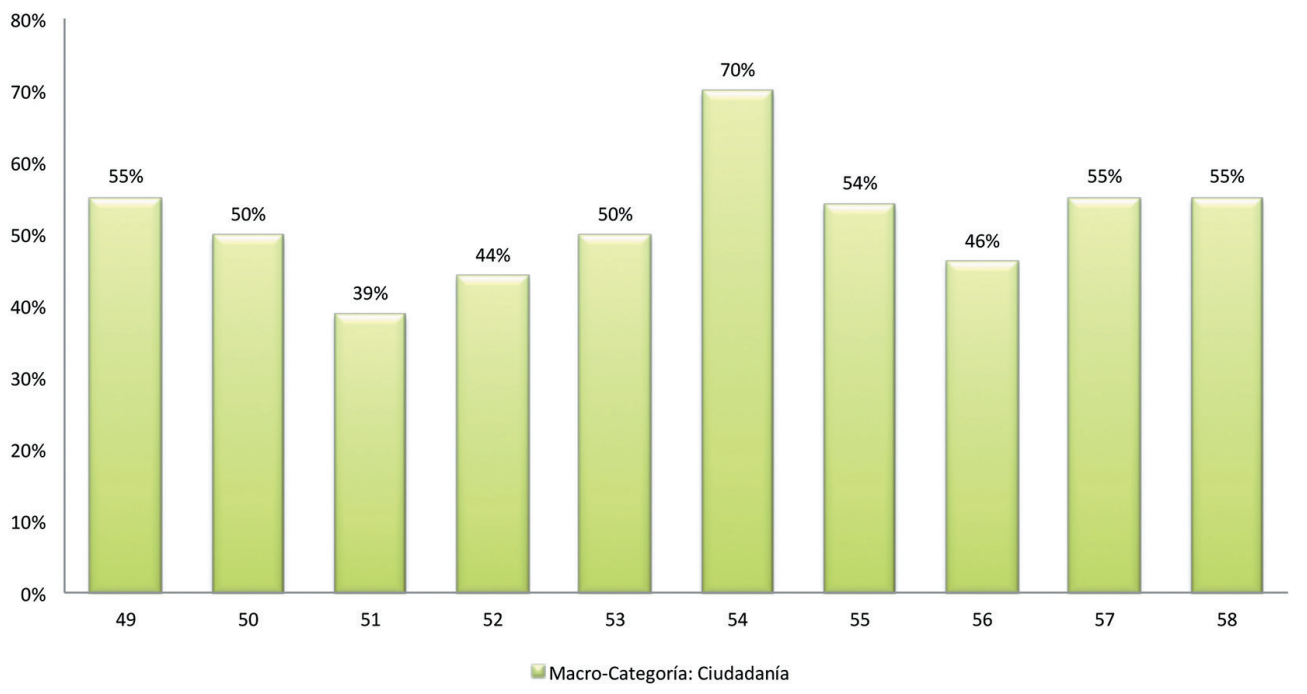

Ser ciudadano o tener ciudadanía significa para la mayoría, el $55 \%$ tener derecho a elegir a los funcionarios que lo gobiernan. $Y$ el $12 \%$ piensa, que el ciudadano es el que tiene el poder para gobernar el país.

El $50 \%$, cree que la cualidad más importante de un ciudadano es su voluntad para defender sus derechos y cumplir con sus deberes. El $31 \%$ opina que la cualidad más importante del ciudadano es el conocimiento de las leyes que rigen ese sistema.
La calidad del ciudadano en Colombia fue evaluada por los estudiantes de la siguiente forma:

Tabla 4. calidad del ciudadano en Colombia

\begin{tabular}{cc}
\hline PORCENTAJE & CONCEPTO \\
\hline $39 \%$ & Satisfactorio \\
$32 \%$ & Deficiente \\
$22 \%$ & Bueno \\
$3 \%$ & Excelente \\
$4 \%$ & Fracasado \\
\hline
\end{tabular}


El $39 \%$ cree que la calidad de ciudadano que impera en nuestra sociedad es satisfactoria, pero dicha elección contrasta con el $32 \%$ que piensa que dicha calidad ya no estan alta sino deficiente, e indica que la percepción sobre este aspecto no está definida por parte de los participantes; algo que si se puede deducir de las elecciones realizadas es que para la mayoría de los encuestados, en Colombia la calidad de ciudadanos excelentes es reducida, pues solo el $3 \%$ considera que esta opción sea la imperante.

Según el $44 \%$ la manera más efectiva que tenemos los ciudadanos para ejercer poder en el gobierno de este país es mediante nuestro voto. Solo el $9 \%$ cree que dicha participación se hace organizándonos en comunidades o en grupos de interés.

El $50 \%$ de los estudiantes entiende a la sociedad civil como las organizaciones por medio de las cuales los ciudadanos buscan entender y solucionar problemas sociales que les afectan y que el Estado y los partidos tradicionales no logran resolver. EI $22 \%$ cree que la sociedad civil son las asociaciones y agrupaciones cuya actividad civil no está controlada por el Estado.

El $70 \%$ piensa que la existencia de una sociedad civil fuerte en nuestro país es importante para consolidar la democracia, pues aumenta la participación del pueblo, el $14 \%$ la considera nociva a la consolidación de la democracia pues debilita el poder de los partidos políticos.

Para el 54\% la existencia de una sociedad civil fuerte en nuestro país ha jugado un importante papel en la defensa de los derechos civiles y/o humanos. El 14\% de los estudiantes considera que la existencia de una sociedad civil fuerte ha jugado un papel importante en la protección de la propiedad privada.
En el $46 \%$ la idea que mejor expresa lo que entiendo por clases sociales es aquella que las define como agrupaciones de seres humanos que tienen en común una misma posición y prestigio social; y el $22 \%$ considera que son aquellas agrupaciones de seres humanos que tienen en común su relación de propiedad o no propiedad con los medios de producción de un país.

Según el $55 \%$ el concepto de clase social es importante porque permite entender muchos de los conflictos que se dan en la sociedad; solo el $13 \%$ considera que las clases sociales son importantes porque permiten entender la razón de ser de muchas leyes que se aprueban en nuestros países.

Sobre las relaciones entre clases sociales y democracia, el $55 \%$ cree que las diferencias de clase afectan a la democracia pues los que tienen más poder económico dominan la política. Solo el $6 \%$ opina que en la democracia debe dominar la clase más preparada para gobernar y en la misma proporción, $6 \%$, opinan que en la democracia debe dominar la clase mayoritaria, que son los trabajadores.

\section{Hallazgos y Conclusiones Preliminares}

Los hallazgos que se presentan a continuación, se realizan a la luz del marco conceptual del proyecto, que gira en torno a las aproximaciones teóricas de autores como A. Cortina, M. Nussbaum y C. Mouffe, entre otros. Con respecto a las tres categorías por las que se indagó en el estudio y el análisis realizado a los hallazgos estadísticos obtenidos por el mismo, surge la siguiente reflexión a modo de conclusiones preliminares, sobre la forma como son percibidos estos aspectos propios de la cultura política y la ciudadanía: 


\section{Con respecto a la Política:}

La percepción que se vislumbra desde la mayoría de los jóvenes universitarios encuestados sobre este concepto es muy parecida al ideal histórico construido desde la antigüedad, donde se entiende a la política como una práctica de todos para el beneficio de la colectividad, se busca el bien común y no los intereses individuales de los más favorecidos; a partir de lo anterior, se define como positiva la forma de percibir la política desde la teoría por parte de los participantes; ahora bien, la percepción cambia significativamente, cuando se cuestiona, ya no sobre las teorías de la política, sino sobre su realidad, en este ámbito, la política es percibida de manera negativa, describiéndose como una actividad por medio de la cual se benefician unos pocos; visión totalmente opuesta a lo planteado y aceptado de la teoría que respalda el accionar político de los individuos; del mismo modo, se cree entonces, que se deben tomar medidas efectivas por parte de todos los sectores de la sociedad, especialmente de los jóvenes y la Universidad, para cambiar las formas tradicionales de hacer política en el país y contribuir, en el caso de las instituciones de educación superior, en la formación no partidista de mejores ciudadanos.

Algo similar ocurre con respecto al poder que se ejerce o se obtiene en el ámbito político y la manera en que se percibe, pues, se presenta la misma contradicción, por una parte, el poder se entiende como una facultad que se otorga por la comunidad para ser gobernada, y por otro, con respecto a la práctica, se piensa que el poder político se obtiene por medio de recursos económicos $y$, por tal razón, los que más gozan de esta facultad en el país son los empresarios, en contraste con la clase trabajadora, que no detenta una mínima parte de dicho poder, según lo percibido por los jó- venes universitarios. De acuerdo a esto, se encuentra una percepción negativa de las prácticas políticas del país, que se caracterizan según los encuestados por su manejo monopolístico, excluyente y poco benéfico para la colectividad.

\section{Con respecto a la Democracia}

Con respecto a este concepto, se encontró que los jóvenes participantes se inclinan en su mayoría por mantener los marcos legales mínimos que garanticen el desarrollo pleno del sistema democrático, pues plantean la necesaria existencia de principios propios de la teoría democrática liberal como lo son:

- La selección libre por parte del pueblo o los ciudadanos de sus representantes en el gobierno.

- Educación para el pueblo para que pueda ejercer su poder.

- La necesidad de un sistema de leyes que garantice la soberanía del pueblo.

- Satisfacción de las necesidades básicas de todos los ciudadanos.

A partir de lo anterior e interpretado desde el marco teórico desarrollado, se observa que las percepciones sobre la democracia que imperan entre los encuestados tiende a mantener una amplia relación con la teoría que define a la democracia como "Democracia Formal" o "Gobierno del Pueblo" concepción que consiste en distribuir el poder político efectivamente entre la mayor parte de los ciudadanos; a su vez, estas percepciones halladas mantienen estrecha relación con el otro sentido teórico que define a la democracia como "Democracia Material", pues según los encuestados, este sistema de gobierno se caracteriza por permitir que el pueblo no tome las decisiones que le atañen sino que elija a sus representantes para que decidan 
por él, se recuerda que la mayoría de los estudiantes opinó que las característica más importantes de este sistema político es que el pueblo o los ciudadanos "Seleccionen libremente a los que los representaran en el gobierno", aunque en el fondo, encontramos poca o escasa confianza en los partidos políticos que agrupan a dichos representantes pues un escaso grupo de los encuestados cree que "en los partidos políticos encontrarán diversas alternativas de selección".

Por otra parte, según los resultados de la investigación, la democracia se fundamenta a partir de un sistema de leyes que garantiza la soberanía del pueblo, percepción que se asimila desde la teoría que define a los sistemas democráticos de gobiernos como capaces de proporcionar las condiciones en las que se pueden desarrollar plenamente las libertades y los derechos del hombre, tal y como la perciben un grupo significativo de los encuestados, que manifiestan que se necesita que "haya un sistema de derecho (Leyes) que garantice que el poder esté en manos del pueblo", se observa que frente a esta percepción que relaciona a la democracia con las normas legales que garantizan su funcionamiento existe una considerable cantidad de encuestados que la perciben de esta manera.

En el sentido de la democracia percibida como el sistema de gobierno que garantiza la satisfacción de las necesidades básicas de todos los ciudadanos, encontramos una percepción estrechamente ligada a las teorías de la democracia dentro del Estado Social de Derecho o Estado de Bienestar, pues al igual que los participantes, que creen que la finalidad más importante de un sistema político democrático es "garantizar la satisfacción de las necesidades básicas de todos los ciudadanos", para algunos teóricos, la democracia se percibe como el sistema que permite de manera amplia y equitativa la satisfacción de dichas necesidades.

\section{Con respecto a la Ciudadanía}

Para los participantes del estudio, el hecho de pertenecer a una comunidad política que les ha otorgado una serie de derechos y deberes para la convivencia, hace posible adquirir la calidad de ciudadano en una sociedad como la nuestra; pero, las tendencias teóricas contemporáneas sobre el tema son enfáticas al resaltar la idea de que no sólo la posesión de derechos y deberes hacen al ciudadano y proponen nuevas formas de ejercer la ciudadanía desde una perspectiva mucho más dinámica. Ahora bien, según los participantes, elegir los funcionarios que los gobiernen, hace del individuo un ciudadano dentro del sistema democrático, se relega así a un solo derecho constitucional toda la dimensión de la ciudadanía. Esta percepción se relaciona con la concepción sobre "Modelo Cívico" o "Ciudadano Cívico" definida por Deicy Hurtado y Gloria Naranjo, para quienes esta forma de definir la ciudadanía como una mera “participación ciudadana amparada en las normas constitucionales del país" goza de una amplia influencia occidental

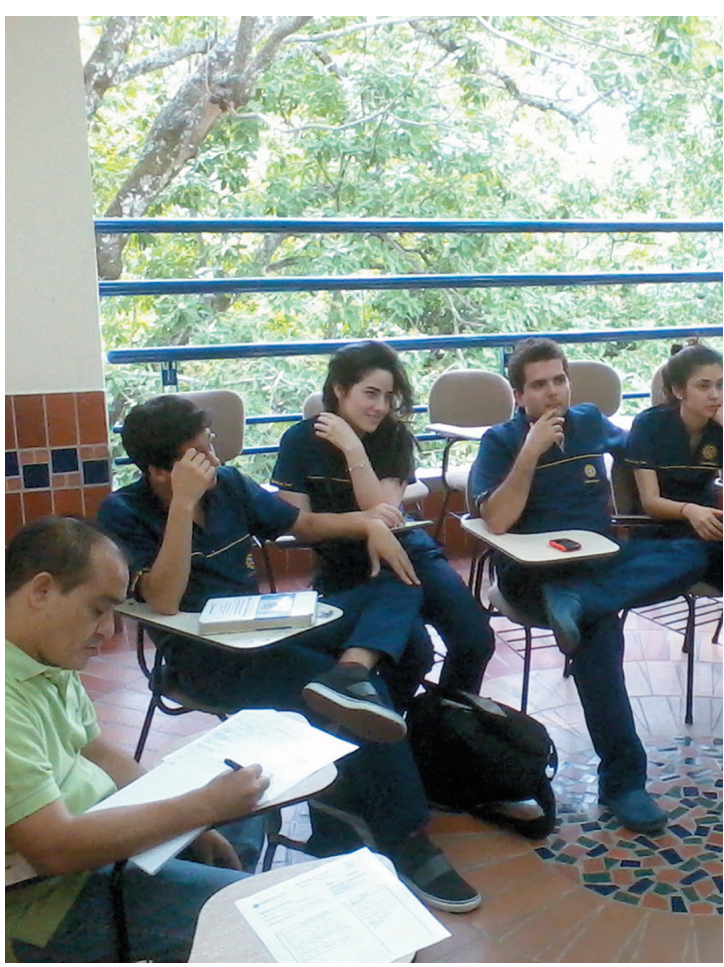




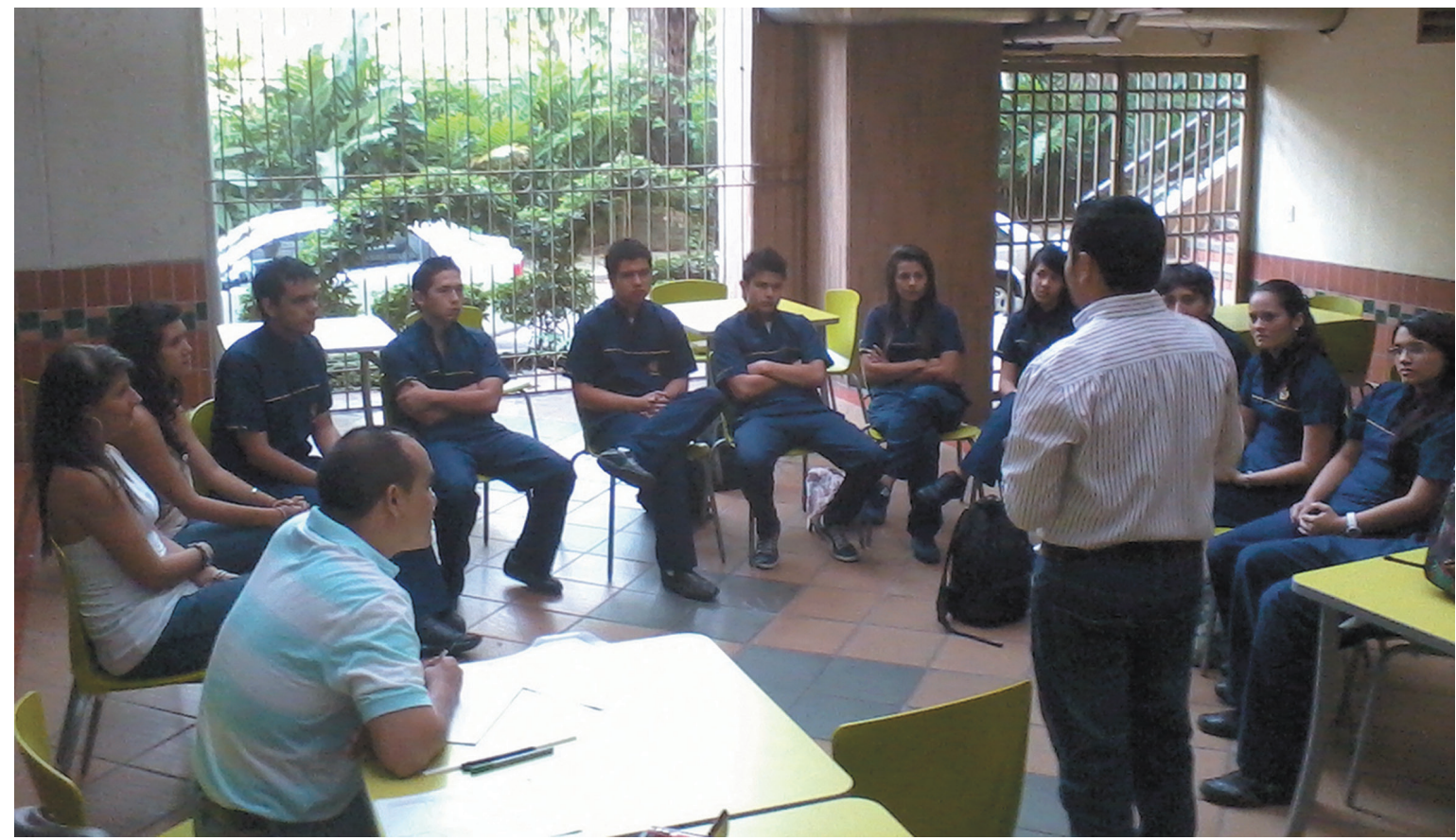

y no permite dar píe a otras formas de comprender la ciudadanía, ya no desde un ideal o "Modelo Cívico", sino desde las mismas ciudadanías existentes que permitan la coexistencia de diversas moralidades públicas y ciudadanías democráticas para avanzar hacia la construcción de nuevos referentes.

De esta manera, entonces, se busca proponer que el punto de partida sean las ciudadanías realmente existentes y no el ideal de ciudadano según el modelo cívico, para avanzar hacia la construcción de unos nuevos referentes interpretativos que, con una propuesta de nuevas pedagogías ciudadanas, se desplace hacia el lugar de la acción política democrática. Dicha concepción constituye entonces al ciudadano como un sujeto que ejerce sus derechos, con capacidad crítica para asumir responsablemente la actuación en la resolución colectiva de los problemas sociales (Hurtado y Naranjo, 2002, 60-64).

En cuanto a la calidad del ciudadano en el país y frente a lo referenciado en el marco teórico sobre las teorías plantadas por Adela Cortina en relación con la percepción ateniense de la ciudadanía, donde el "buen ciudadano es el que con su participación política no busca nada diferente al bien común", se encuentra una significativa diferencia, pues contario a lo planteado por Cortina sobre el ideal de ciudadano ateniense, los estudiantes tienen la percepción de que los miembros de la comunidad democrática gozan de una calidad satisfactoria y buena de la ciudadanía, porque se basan en el ejercicio del derecho al voto y en la exigencia de sus derechos para evaluar dicha calidad, más no se tiene en cuenta si dichas acciones van en busca del Bien Común; en cambio, esta percepción si guarda estrecha relación con la concepción romana de ciudadano descrita por Cortina, donde ciudadano es quien actúa bajo la ley y espera la protección de la misma dentro del imperio, que hace ver a la Ciudadanía sólo como una condición jurídica y no como una exigencia de implicación política como en Atenas; en pocas palabras, la ciudadanía es una plataforma jurídica que sirve para reclamar derechos, pero no exige responsabilidades frente al Estado. Se percibe entonces una gran problemática de información y aplicación de las normas Constitucio- 
nales y organizaciones de la sociedad civil que permiten el ejercicio del poder político por medios diferentes al voto.

De parte de la misma autora, encontramos que la definición de ciudadano planteada por Thomas $\mathrm{H}$. Marshall se relaciona en parte con lo que percibe la mayoría de los encuestados sobre tema, pues para Marshall se debe considerar ciudadano a aquel que en una comunidad política goza no sólo de derechos civiles (libertades individuales), en los que insisten las tradiciones liberales, no sólo de derechos políticos (participación política), en los que insisten los republicanos, sino también en derechos sociales (trabajo, educación, vivienda, salud, prestaciones sociales en tiempos de especial vulnerabilidad) (Cortina, 2005, 66) y para la mayoría de los participantes, ser ciudadano es ser "miembro de una comunidad política en la que se tiene una serie de derechos y deberes".

La Ciudadanía, por su parte, goza de una percepción favorable, pero limitada, dentro de los jóvenes, pues para los participantes, ser ciudadano obedece solamente a la posesión de deberes y derechos otorgados por la sociedad para la vida en convivencia, y se entiende como deber y derecho principal, la elección de los funcionarios que lo gobiernan dentro de una sociedad con altos índices de corrupción por parte de sus representantes y partidos políticos. Para ello se propone, por parte de los encuestados, la creación de nuevos espacios y ambientes políticos donde se puedan generar formas justas y modernas de hacer política, la creación de nuevos ideales y grupos que representen los intereses y las necesidades de la comunidad.

Por las anteriores razones, se hace necesaria la continuidad de este tipo de investigaciones, que permitan el acercamiento desde la academia a los problemas de la vida en sociedad y a la realidad política que viven los jóvenes en el país, con el fin de comprender dichos fenómenos en mayor medida y tener la posibilidad de replantearlos con vista a un mejor futuro, recordemos la vieja frase, que siembra la gran responsabilidad en los jóvenes, de tener que tomar el timón de la sociedad en un tiempo no muy lejano. Para esto, desde la academia, específicamente desde las humanidades, se debe plantear un aporte significativo a los marcos educativos que pretenden la formación de profesionales capaces de transfigurar la oscura realidad política que perciben en su sociedad, con el firme propósito de generar nuevas formas de ejercer la democracia y la ciudadanía $y$, por ende, una nueva forma de construir sociedad.

\section{Referencias}

Castillo E. (2003). Democracia y Ciudadanía en la escuela colombiana. Acción pedagógica. Vol.12 No. 1

Cardona, R. (2010) Imaginarios de Ciudadanía, de la Ciudadanía restrictiva a la Ciudadanía Planetaria. Sobre los imaginarios de ciudadanía en algunos estudiantes Universitarios. Editorial USTA, Bucaramanga. ISBN: 978-958-8477-13-8

Cortina, A. (2001) Alianza y Contrato. Política, Ética y Religión. Trotta. Madrid.

Cortina, A. (1997) Ciudadanos del mundo. Hacia una teoría de la ciudadanía. Editorial Alianza, Madrid.

Cortina, A. (1996) El quehacer ético, guía para la educación moral. Santillana, Madrid.

Hoyos, G. (2008) (Memorias). “Crisis, Filosofía y Nuevo Humanismo: Fenomenología y Teoría Crítica de la Sociedad". IX Congreso Internacional de Humanidades: Biodesarrollo. Universidad Santo Tomás, Bucaramanga.

Hoyos, G. Serna, J. Gutiérrez, E. (2007) Borradores para una filosofía de la educación. Siglo del Hombre Editores, Bogotá.

Hoyos, G. (1995) Ética y Educación, para una ciudadanía democrática. Bogotá. Editorial SMD.

Hoyos, G. (1995) Ética y Educación, para una ciudadanía democrática. Universidad Nacional. Bogotá.

Hurtado D., Naranjo Gloria. (2002) "Aprendizajes Sociales y Pedagogías Ciudadanas". Universidad de Antioquia. Instituto de Estudios Políticos. En: Encuentro Distrital de Experiencias en Educación para la Democracia. Bogotá, octubre 10 y 11 de 2002. Pp. $60-64$

Maturana, H. (2002) El sentido de lo humano. Dolmen Ediciones, Santiago, España.

Mejía, R. (2006) Educación(es) en la(s) globalización(es) entre el pensamiento único y la nueva crítica. Editores Desde Abajo, Bogotá.

Mejía, M; Awad G. M. (2004) Educación Popular hoy En tiempos de globalización. Ediciones Aurora, Bogotá.

Mouffe, C. (1999) El Retorno de lo Político. Comunidad, ciudadanía, pluralismo, democracia radical. Paidós. Barcelona. 
Nussbaum, M. (2007) Las fronteras de la justicia. Consideraciones sobre la exclusión. Paidós, Barcelona.

Nussbaum, M. (2005) El cultivo de la humanidad. Una defensa clásica de la reforma en la educación liberal. Paidós, Barcelona.

Nussbaum, M (2003) La terapia del deseo. Teoría y práctica en la ética helenística. Paidós, Barcelona.

Ospina, H. Alvarado, S. Compiladores (1998) Ética ciudadana y derechos humanos de los Niños. Una contribución a la paz. Cooperativa Editorial Magisterio, CINDE, Bogotá.

Ospina, W. Columna El Espectador, Domingo 6 de septiembre de 2009, recuperado de http://www. elespectador.com/columna159750-educacion el 7 de septiembre de 2009

Rawls, J. (1978) Teoría de la justicia. Fondo de Cultura Económica, Madrid.

Revista Temas (2011) Vol. III, No. 5. “Del concepto de ciudadanía: una aproximación teórica". Universidad Santo Tomás: Bucaramanga. Pp. 93-110.

Sen A. (2007) Primero la gente: una mirada desde la ética del desarrollo a los principales problemas del mundo globalizado. Ed. Deusto, Barcelona.
Sen A. (2000) Desarrollo y libertad. Planeta, Barcelona.

Sen, A. (1999), Nuevo examen de la desigualdad. Alianza editorial. Madrid.

Rodríguez, José Gregorio (Editor). 2004. Rutas pedagógicas de la historia en la educación básica de Bogotá. Bogotá: Universidad Nacional de Colombia-Programa RED-Instituto de Investigación Educativa y Desarrollo Pedagógico IDEP. Tomado de: http://www.javeriana.edu.co/ Facultades/C Sociales/universitas/documents/vivas.pdf Noviembre 13-08

Urquijo Angarita, Martín Johani (2008) La Libertad como Capacidad El enfoque de las capacidades de Amartya Sen y sus implicaciones en la ética social y política. Programa Editorial Universidad del Valle, Santiago de Cali.

Urquijo Angarita, Martín Johani. (2007a) El enfoque de las capacidades de Amartya Sen: Alcances y límites. Tesis doctoral de la Universidad de Valencia, España, dirigida por Adela Corina Orts. Texto en pdf, de 390 páginas, disponible en la siguiente dirección: http://www. tesisenxarxa. net/TESIS UV/AVAILABLE/TDX-0520108-145406// urquijo.pd $\bar{f}$ (Consultada el 28 de octubre de 2008) 